\title{
AN ALGEBRAIC PROOF OF AN ANALYTIC RESULT OF SHUCK'S
}

\author{
BY L. G. ROBERTS
}

Communicated by Hyman Bass, November 29, 1972

In [1] the authors pose the following problem: Let $F\left(X_{1}, \ldots, X_{r}\right)$ be a polynomial in $r$ variables with integer coefficients. Let $p$ be a prime. For each positive integer $n$ let $c_{n}$ be the number of solutions modulo $p^{n}$ of the congruence $F\left(x_{1}, \ldots, x_{r}\right) \equiv 0 \bmod \left(p^{n}\right)$. Form the Poincare series of $F$

$$
P_{F}(t)=\sum_{n=1}^{\infty} c_{n} t^{n}
$$

Is $P_{F}(t)$ a rational function in $t$ ?

John Shuck [3] generalized this problem and then answered it affirmatively in the "nonsingular" case. His approach was to develop a calculus on manifolds over $p$-adic fields (including a theory of integration on submanifolds, Fubini's theorem, and change of variables), and then to utilize analytic techniques for counting.

In the following we present another reformulation of the problem, this time in an algebraic geometric setting, and give a purely algebraic and quite elementary proof in the smooth case.

My thanks here are to M. J. Greenberg who insisted that this should be written down.

1. A reformulation. We consider the following situation: $S$ is a scheme (that is, a prescheme in the sense of EGA), $X$ is an $S$-scheme, $I$ a quasicoherent ideal of $O_{S}$. Let $S_{n}$ be the subscheme of $S$ defined by the ideal $I^{n}$. Then we have the cartesian diagram

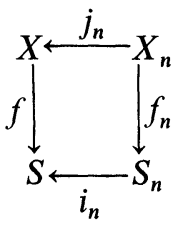

Let $\Gamma_{n}=\operatorname{Hom}_{S_{n}}\left(S_{n}, X_{n}\right)=\operatorname{Hom}_{S}\left(S_{n}, X\right)$. We pose the following problem: If $c_{n}=\operatorname{card}\left(\Gamma_{n}\right)$ is finite for all $n$ greater than some $N$, set

AMS (MOS) subject classifications (1970). Primary 14G05, 14F10.

Key words and phrases. Sections over local schemes, Poincaré polynomial, relative differentials, regular ideal, smooth relative schemes. 


$$
P_{X / S, I}(t)=\sum_{n \geqq N} c_{n} t^{n}
$$

When is $P_{X / S, I}(t)$ rational?

REMARK. We do not take this formulation seriously; we put it down only to give a convenient way of discussing various hypotheses that one might impose. In fact even with $X / S$ smooth, $S$ affine, we must still impose very stringent conditions on $I$ to obtain the rationality.

2. The main result. We will prove the following

THEOREM. Let $X / S$ be smooth, where $S$ is the spectrum of a noetherian ring $A, I$ an ideal of $A$ generated by a regular element $x$ of $A$. Further assume that $A / I$ is finite and that the sets $\Gamma_{n}$ are finite for all $n$ (this is automatic if $X$ is affine, or if $S_{n}$ is local for all $n$ and $X$ is quasi-compact). Then $P_{X / S, I}(t)$ is rational.

REMARK. If the assumptions are as in the theorem, but with $I$ generated by an $A$-regular sequence $x_{1}, x_{2}$, then $P_{X / S, I}(t)$ is not in general rational.

Proof. We first note that the assumptions imply immediately that $A / I^{n}$ is finite for all $n$. The parenthetical remark then follows since in the affine case $X$ is then $\operatorname{Spec}\left(A\left[T_{1}, \ldots, T_{n}\right] /\left(f_{1}, \ldots, f_{r}\right)\right)$, and in the local case every $S$-morphism of $S_{n}$ into $X$ factors through some $U_{i}$ where $U_{1}, \ldots, U_{k}$ is a finite affine cover of $X$.

Now consider the diagram which we shall use consistently:

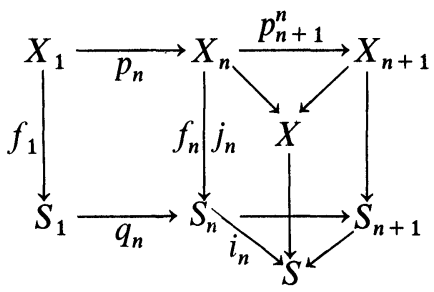

First note that there is a natural map $\alpha_{n}^{n+1}: \Gamma_{n+1} \rightarrow \Gamma_{n}$, induced essentially by composition with $q_{n+1}^{n}: S_{n} \rightarrow S_{n+1}$. Let $\beta_{n+1}: \Gamma_{n+1} \rightarrow \Gamma_{1}$ designate the composition of the corresponding $\alpha_{i}^{i+1}$.

The idea is to study the maps $\alpha_{n}^{n+1}$. Let $J_{n}$ be the ideal of $\operatorname{Spec}\left(A_{n+1}\right)$ $=\operatorname{Spec}\left(\mathrm{A} / I^{n+1}\right)$ which defines the closed subscheme $S_{n}$. Then $J_{n}=\left(I^{n} / I^{n+1}\right)$ and has square zero. Since $X_{n+1} / S_{n+1}$ is smooth it follows immediately from the definition of smoothness [EGA IV, 17.3.1] that $\alpha_{n}^{n+1}$ is onto.

Now fix an element $u$ of $\Gamma_{n}$, and consider the corresponding morphism $u_{0}: S_{n} \rightarrow X_{n+1}$, obtained by composition with $p_{n+1}^{n}$. Let $G$ be the $O_{S_{n}}{ }^{-}$ module $\operatorname{Hom}_{\boldsymbol{O}_{S_{n}}}\left(u_{0}^{*} \Omega_{X_{n+1} / S_{n+1}}^{1}, J_{n}\right)\left(J_{n}\right.$, having square zero, is, in a natural way, an $O_{S_{n}}$-module). Finally (following [EGA IV, 16.5.14]) introduce the sheaf of sets $P$ on $S_{n}$ defined as follows: for every open $U_{0}$ of $S_{n}$ let $U$ be 
the corresponding open set in $S_{n+1}$ (which is well defined since $S_{n}$ and $S_{n+1}$ have the same underlying topological space); let $P\left(U_{0}\right)=$ the set of $S_{n+1}$ morphisms from $U$ to $X_{n+1}$ which agree with $u_{0}$ on $U_{0}$. Now since $S$ is affine, and since $X / S$ is smooth it follows immediately from [EGA IV, 16.5.17 and 16.5.18] that $P$ is, first a $G$-torseur (smoothness), and secondly a trivial $G$-torseur (affineness), that is to say, isomorphic to $G$ (as $G$ torseurs). Hence we obtain that the cardinality of the fibre $\left(\alpha_{n}^{n+1}\right)^{-1}(u)$ is precisely that of $\operatorname{Hom}_{O_{S_{n}}}\left(u_{0}^{*} \Omega_{X_{n+1} / S_{n+1}}^{1}, J_{n}\right)$. Since we are assuming that all the $\Gamma_{n}$ are finite, it follows that this cardinality is also finite.

We now study this module. With $u, u_{0}$ as above, let $v_{0}$ in $\Gamma_{1}$ be the image of $u_{0}$ under $\beta_{n}$. Then we have the

LEMMA. There is an isomorphism

$$
\operatorname{Hom}_{O_{S_{n}}}\left(u_{0}^{*} \Omega_{X_{n+1} / S_{n+1}}^{1}, J_{n}\right) \simeq \operatorname{Hom}_{O_{S_{1}}}\left(v_{0}^{*} \Omega_{X_{1} / S_{1}}^{1}, J_{1}\right) .
$$

Proof. With reference to the diagram above, it follows easily that $p_{n+1} v_{0}=u_{0} q_{n}$, hence $j_{n+1} p_{n+1} v_{0}=j_{n+1} u_{0} q_{n}$, or that $j_{1} v_{0}=j_{n+1} u_{0} q_{n}$. Then we have the sequence of naturat isomorphisms

$$
\begin{aligned}
\operatorname{Hom}_{O_{S_{1}}}\left(v_{0}^{*} \Omega_{X_{1} / S_{1}}^{1}, J_{1}\right) & =\operatorname{Hom}_{O_{S_{1}}}\left(v_{0}^{*} j_{1}^{*} \Omega_{X / S}^{1}, J_{1}\right) \\
& =\operatorname{Hom}_{O_{S_{1}}}\left(q_{n}^{*} u_{0}^{*} j_{n+1}^{*} \Omega_{X / S}^{1}, J_{1}\right) \\
& =\operatorname{Hom}_{S_{S_{n}}}\left(u_{0}^{*} \Omega_{X_{n+1} / S_{n+1}}^{1}, q_{n}^{*} J_{1}\right) .
\end{aligned}
$$

Finally we make the observation that due to the assumptions on $I$ there is a (noncanonical) isomorphism between $I / I^{2}$ and $I^{n} / I^{n+1}$. From this it follows immediately that $q_{n}^{*} J_{1}$ is isomorphic as an $O_{S_{n}}$-ideal to $J_{n}$, and we are finished with the lemma.

We can now complete the proof of the theorem. Let $v_{1}, \ldots, v_{k}$ be the members of $\Gamma_{1}$. Each $\Gamma_{n}$ splits up as a direct sum of sets $\Gamma_{n, i}=\beta_{n}^{-1}\left(v_{i}\right)$, with cardinality $c_{n, i}$ say. Let $c_{n}$ be the cardinality of $\Gamma_{n}$, and $r_{i}$ that of $\operatorname{Hom}_{O_{S_{1}}}\left(v_{i}^{*} \Omega_{X_{1} / S_{1}}^{1}, J_{1}\right)$. Then $c_{n+1, i}=r_{i} c_{n, i}=r_{i}^{n}$. The rationality is then clear, and we find that $P_{X / S, I}(t)=\sum_{i=1}^{k} t /\left(1-r_{i} t\right)$.

3. Remarks. (a) We indicate the nonrationality for (slightly) more general $I$. Say that $I$ is generated by an $A$-regular sequence $x_{1}, \ldots, x_{d}$. Then we know by the quasi-regularity of $I$ that the natural map $(A / I)\left[T_{1}, \ldots, T_{d}\right] \frown \mathrm{Gr}_{I}(A)$ is an isomorphism of graded rings. If, in addition, $\Omega_{X / S}^{1}$ is free of finite rank (it is locally free in any case), one can easily compute the cardinalities of the $\operatorname{Hom}_{O_{S_{n}}}\left(u_{0}^{*} \Omega_{X_{n+1} / S_{n+1}}^{1}, J_{n}\right)$. One finds that if $d \geqq 2$, the Poincaré series can never be rational, the growth of the $c_{n}$ being too rapid.

(b) If $X$ were a group scheme we could by a homogeneity argument 
obtain that the various $\operatorname{Hom}_{\boldsymbol{O}_{s_{1}}}\left(v_{1}^{*} \Omega_{X_{1} / S_{1}}^{1}, J_{1}\right)$ 's are isomorphic to the one corresponding to the unit section.

(c) The germ of the above argument is contained in [2, $\$ 19$, Proposition 20] and, in fact, the result given there can be utilized to give another proof of the above in the setting studied by Néron (essentially affine varieties over $p$-adic bases).

\section{BIBLIOGRAPHY}

1. Z. Borevich and I. Shafarevich, Number theory, "Nauka", Moscow, 1964; English transl., Pure and Appl. Math., vol. 20, Academic Press, New York, 1966. MR 30 \# 1080; MR 33 \#4001.

EGA IV A. Grothendieck, Éléments de géométrie algébrique. IV. Étude locale des schemas et des morphismes de schemas. IV. Rédigés avec la collaboration de J. Dieudonné; Publ. Math. de l'I.H.É.S., no. 32, Presses Universitaires de France, Vendôme, 1967. MR 39 \# 220.

2. A. Néron, Modèles minimaux des variétés abéliennes sur les corps locaux et globaux, Publ. Math. de l'I.H.É.S., no. 21, Presses Universitaires de France, Vendôme, 1964. MR 31 \# 3423.

3. J. Shuck, Calculus on p-adic manifolds, with applications to number theory, Thesis, Northeastern University, 1969.

Department of Mathematics, University of British Columbia, Vancouver, British Columbia, Canada 